\title{
LOS REGLAMENTOS PARLAMENTARIOS Y EL ORDENAMIENTO JURIDICO
}

\author{
POR \\ CLARO-JOSE FERNANDEZ-CARNICERO GONZALEZ \\ Letrado de las Cortes
}

\begin{abstract}
SUMARIO
I. EL. REgLAMENTO PARLAMENTARIO, EXPRESIÓN DE LA NORMATIVIDAD INSTITUCIONAL DEL ESTADO. QUIEBRA DE LA CONCEPCIÓN FORMALISTA DE LAS FUENTES DEL DERECHO.-II. El REgLAMENTO PARLAMENTARIO Y EL ORDENAMIENTO JURÍDico. ARTICULACIÓN FUNCIONAL DE LOS «INTERNA CORPORIS» Y DE LOS «EXTERNa CORPORTS».-III. Parlamento Y Derecho. El DeRECHO PARLAMENTARIO COMO PRESUPUESTO Y LÍMITE DE LA AUTONOMÍA DE LAS CÁMARAS. UNa aPROXIMACIÓN MÁS A LA CRISIS DE LA INSTITUCIÓN PARLAMENTARIA.

«Le droit est expression de 'principes', donc de 'règles'; il est lié à la notion de 'règlementarité'. Sa tâche consiste à faire ressortir ce qui est valable, ce qui, par-delà la 'normalité', est normativité.»

«L'acte politique (l'acte d'organisation) par excellence est celui qui définit les procédures.»

«Mais cela même démontre que l'acte politique est soumis au principe de normativité, qu'il doit obéir au droit, qu'il n'en est pas le maître.»

(H. A. Schwarz-Liebermann, Les dimensions 'du droit.)

«La storia del nostro secolo rivela, sotto il profilo delle vicende legislative, un radicale indirizzo centrifugo.»
\end{abstract}

(Natalino Irti, L'età della decodificazione.)

\section{I}

Sigue siendo común entre los juristas hacer gala de la permanencia y refinamiento conceptual del Derecho privado, primariamente fiel a la tradición jurídica romana, frente al relativismo y, por consecuencia, a la movilidad de los principios que presiden el devenir y el ser del Derecho público. Y aun dentro de este segundo dominio normativo, los cultivadores del Derecho 
político ven cómo día a día el objeto de su disciplina —el régimen jurídico de los poderes públicos y de los grupos a través de los cuales se articulan las fuerzas sociales - cede terreno a los expertos en Derecho administrativo, mejor pertrechados en cuanto al rigor técnico de sus presupuestos doctrinales. Debe reconocerse, sin embargo, y sin regatear méritos donde los haya, que esta mayor auctoritas se ve favorecida por el hecho de que la Administración pública y los ciudadanos afectados por su acción resultan siempre más próximos que el Estado.

Tras ese flash de la actitud de la doctrina se esconde una evidencia: los juristas no creen en «el Derecho», creen sólo en las instituciones. En otros términos, y siguiendo a Santi Romano, la función estabilizadora del Derecho no puede menos de ser institucional, es decir, tendente a organizar entes sociales conforme a un postulado de permanencia. Instrumento, y no el único, de esa función es la norma jurídica o mandato jurídico con eficacia social organizadora, en expresión de De Castro.

Basta ese marco conceptual para asumir el contenido normativo de los reglamentos parlamentarios y, por ende, su carácter de instrumento de acción institucional del Parlamento mismo.

En nuestra doctrina, Pérez Serrano vincula el reglamento parlamentario al fenómeno jurídico de la normación autonómica o estatutaria; fruto en este caso de la virtualidad del principio de autonomía en el campo del Derecho público, paralelamente a la desplegada en el Derecho privado.

A nuestro juicio, el principio de autonomía, al ser determinante de la articulación del régimen interno de una Cámara legislativa, es expresión directa de la vis instituyente ( $\mathrm{y}$ no tanto del poder constituyente, como entendieron Sieyès y Mirabeau) con que se manifiesta el principio de soberanía del Estado. Esta es la razón de que se reconozca al Parlamento el carácter de órgano o institución constitucional, a través del cual se vincula al Estado la representación política de la nación o del pueblo.

El primer problema con que se tropieza el estudioso del Derecho parlamentario al tratar de reflexionar sobre la naturaleza jurídica de los reglamentos de las Cámaras no es tanto el de la superficial equivocidad del término «reglamento» cuanto el del valor primario y nuclear del concepto de ley, manifestación a su vez de la formalización sistematizadora de las fuentes del Derecho.

Es evidente que la primacía normativa de la ley se ha traducido en la doctrina elaborada sobre el reglamento parlamentario en una definición negativa de éste, por contraste con las notas que se predican de aquélla.

Así, frente a la concepción de la ley como mandato subjetivo externo (de un órgano estatal que, como advierte Santi Romano, desde su posición de supremacía impone su voluntad a sus subordinados), se pone de relieve el alcance meramente interno del reglamento parlamentario. El equívoco de esta concepción imperativista del Derecho, nos dirá el gran maestro italiano, consiste en haber configurado como destinatarios a aquellos respecto de los cuales la ley produce, directa o indirectamente, consecuencias jurídicas.

Dentro de la doctrina germánica, encontramos en Hatschek una formulación ya clásica del concepto negativo de reglamento parlamentario, sometido al de la ley como prototipo normativo único y excluyente. Así, dicho autor enumera 
respecto de aquél los presupuestos formales o de eficacia externa omitidos: no es objeto de sanción, promulgación ni publicación, sólo está en vigor durante la legislatura para la cual fue aprobado (acogiendo la teoría radical del poder constituyente antes aludida) y, por último, su eficacia interna impide que pueda ser invocado ante los tribunales. Desde la doctrina italiana, Bon Valsassina añadiría dos notas, igualmente por vía de negación, desde la teoría de la norma-mandato: no confiere facultades ni crea obligaciones.

La conclusión de ese animus dialéctico, por explicitarse casi en la propia argumentación, es obligada. El reglamento parlamentario, nos dirá Hatschek, es un mero cuerpo de reglas convencionales, es decir, preceptos fácticos sin valor jurídico, al no formar parte de las fuentes del Derecho.

Intentemos llegar a una definición positiva del reglamento de las Cámaras a través de los propios preceptos constitucionales.

El artículo 72 de la Constitución española vigente dispone en su apartado 1 que «Las Cámaras establecen sus propios Reglamentos, aprueban autónomamente sus presupuestos y, de común acuerdo, regulan el Estatuto del Personal de las Cortes Generales. Los Reglamentos y su reforma serán sometidos a una votación final sobre su totalidad, que requerirá la mayoría absoluta»; precepto este que debe concordarse con el artículo $10^{\circ}$, sección V.2 de la Constitución de los Estados Unidos, con el artículo 64 de la Constitución italiana y con los artículos 40.1 y 52.3 de la Ley Fundamental de Bonn, entre otros.

Reglamento, presupuestos y personal son expresión de la autonomía institucional del Parlamento. Como diría Mirabeau, la Asamblea, al representar a la nación, actúa como juez y árbitro de sí misma.

El reglamento parlamentario, como norma de organización, consagra un procedimiento cuyo objeto es garantizar la plenitud de expresión de la voluntad de la Cámara. Todo procedimiento parlamentario, advierte Longi, debe dirigirse ante todo, a permitir la expresión genuina y libre, aunque de modo ordenado, de la voluntad de las Cámaras.

En suma, el reglamento parlamentario es una norma condicionante de la virtualidad institucional del Parlamento. Bástenos aludir a las remisiones que a él hace la Constitución para la determinación del régimen de la iniciativa legislativa (art. 87.1), de la tramitación de las proposiciones de ley (art. 89.1), de fijación de mayorías especiales (art. 79.2), del establecimiento de un tiempo mínimo para debatir interpelaciones y preguntas (art. 111.1), del régimen de acuerdos de declaración del carácter secreto de las sesiones parlamentarias de las Cámaras (art. 80) y de un procedimiento especial y sumario para el control de los decretos-leyes (art. 86.2).

Con el carácter de norma excepcional, la Constitución prevé un Reglamento de las Cortes Generales para los supuestos de sesiones conjuntas de ambas Cámaras (art. 72.2).

El Reglamento de las Cámaras, sin embargo, es, antes que norma condicionante, norma condicionada; es decir, antes que condición, efecto. Efecto de la preexistencia de la institución parlamentaria, de la que recibe su justificación. Sólo a partir de esa prefiguración institucional el reglamento parla- 
mentario se vincula directamente a la Constitución; y es desde esta vinculación como debe entenderse la autonomía normativa interna de las Cámaras.

De otra parte, si bien el reglamento parlamentario es expresión del autogobierno de las Cámaras, ello no supone que asuma la función de garantizar la independencia de éstas. Tal independencia constituye el presupuesto que debe hallarse consagrado en la Constitución y una realidad que debe materializarse en la dialéctica real de los poderes del Estado. En otros términos: garantizar la inmunidad de los parlamentarios, objeto propio de la norma reglamentadora, no equivale a preservar la autonomía institucional del Parlamento.

Hasta aquí el valor o significado político del reglamento parlamentario. Acerquémonos ahora a su estricta dimensión de norma jurídica.

Es evidente que concurren en los Reglamentos de las Cámaras los caracteres de auctoritas (en razón del poder jurídico o competencia del que dicta la regla; competencia articulada, por otra parte, en los mismos términos de rigidez formal que en el caso de las leyes orgánicas, como se desprende del artículo 81.2 de la Constitución), ratio (o contenido intrínseco de dicha regla) y effectus (o realidad social que al ser asumida le confiere validez), notas que la doctrina suele verificar en toda norma positiva. La estructura de ésta (situación de hecho regulada y eficacia jurídica, ordinaria o, en caso de incumplimiento, extraordinaria, determinante, como veremos después del control de constitucionalidad) concurre igualmente en la .regla que comentamos.

En el terreno de los principios resulta, por tanto, difícil encontrar un motivo lógico para excluir al reglamento parlamentario del universo de las normas jurídicas. Pero la lógica es compañera difícil del jurista.

El más somero examen de la evolución de la teoría general del Derecho, y en concreto de las doctrinas sobre la norma jurídica, nos suministra tres órdenes de razones que explican la supuesta bastardía conceptual del reglamento parlamentario.

En primer lugar, la primacía histórica de las normas jurídicas de acción sobre las de organización, que llevó incluso en determinadas circunstancias a negar el carácter jurídico a las segundas.

En segundo lugar, la rigidez formalista y formalizadora del enunciado de las fuentes de un ordenamiento jurídico; rigidez ordinariamente consagrada en los Códigos Civiles, nutridos del espíritu centrípeto, limitado y estable de la burguesía liberal. La revisión de esa pretensión excluyente se mira con reparos, ya que, formalmente al menos, supone la puesta en tela de juicio del principio de jerarquía normativa que de ordinario sigue a aquel enunciado (es el caso de los apartados 1, 2 y 3 del artículo $1 .^{\circ}$ del Código Civil).

En tercer lugar, y siguiendo en el horizonte del Derecho europeo continental, la crisis del principio de generalidad de la ley, presupuesto mismo de todo el movimiento racionalista codificador, ha afectado a la virtualidad institucional del reglamento parlamentario, que, al fin y al cabo, a diferencia del Derecho inglés, traduce respecto del régimen interno de las Cámaras aquel presupuesto.

En el constitucionalismo de la segunda posguerra, como advierte Natalino Irti en reciente ensayo, la ley pasa de ser una regla instrumental de alcance 
general a ser una regla final de contenido especial. A la edad ochocentista de los Códigos liberales sigue la época de las leyes especiales.

Dentro del Parlamento, la crisis del principio de generalidad de la ley alcanza al sentido mismo o función institucional del Reglamento de las Cámaras; de abstracta regla del juego parlamentario pasa a ser el marco mínimo dentro del cual los partidos políticos representados pactan a corto plazo.

De otra parte, la asunción por la ley de las características de concreción e individualidad propias del negocio jurídico privado ha venido favorecida por cuanto que en la génesis institucional de la ley como norma ordinaria antes que una voluntad parlamentaria articulada (fruto, por tanto, de un debate abierto) ha operado en cada caso el pacto de los estados mayores o portavoces de los partidos.

Como vemos, en este tercer orden de consideraciones aflora, una vez más, la vieja tensión dialéctica entre la jurisprudencia de conceptos y la jurisprudencia de intereses; tensión que sólo desde la tópica de la institución parlamentaria, es decir, aproximando sus posibilidades operativas a la posición que constitucionalmente ocupa, puede resolverse. Así, desde esta premisa, que nos atrevemos a postular como la única fecunda en la renovación del Parlamento, entendemos que debiera reflexionarse sobre la trascendencia que la estabilidad del reglamento, al igual que los Standing Orders en el Derecho anglosajón, tiene para el prestigio y la virtualidad institucional de aquél. En otros términos: el valor jurídico de ese reglamento está en función de la naturaleza y el alcance que se reconozca a los actos parlamentarios que tienen lugar a partir de su adopción. En la medida en que se confunda la voluntas del acto con la ratio normativa del reglamento parlamentario, éste pierde su validez.

A partir del valor de estabilidad institucional, el reglamento parlamentario se integra como norma, primariamente y con rechazo por estéril de toda pretensión clasificadora, en el fenómeno de lo jurídico; fenómeno que, como reconoce la tan citada exposición de motivos de la vigente Ley Reguladora de la Jurisdicción Contencioso-Administrativa de 27 de diciembre de 1956, «no se encierra y circunscribe a las disposiciones escritas, sino que se extiende a los principios y a la normatividad inmanente en la naturaleza de las instituciones».

\section{II}

Toda consideración crítica de la definición jurídico-positiva del ordenamiento jurídico, como haz de normas vigentes en un Estado concreto, obliga a quien la asume a tomar posición sobre la materia lo más explícitamente posible.

La Constitución española, como suprema definición normativa del Estado, concibe el ordenamiento jurídico necesariamente en términos de estabilidad; así, en su artículo 1, apartado 1, sitúa al Estado en la posición de propugnar «como valores superiores de su ordenamiento jurídico la libertad, la justicia, la igualdad y el pluralismo político». La norma fundamental asume así la 
función dogmática de ser idea motriz de ese ordenamiento; pero no lo define $y$, por tanto, se subsume en el mismo como en una realidad preexistente. Esto se ve confirmado por el tenor del artículo 9, apartado 1, conforme al cual: «Los ciudadanos y los poderes públicos están sujetos a la Constitución $y$ al resto del ordenamiento jurídico.»

Es el Código Civil el que, por la vía de la enumeración de las fuentes del ordenamiento jurídico español, define a éste, reconociendo como tales en su artículo $1 .^{\circ}$, apartado 1 , a «la ley, la costumbre y los principios generales del Derecho». Una vez más, se pone de manifiesto la preeminencia jurídicomaterial de los preceptos de dicho cuerpo legal, muy por encima de su rango normativo. Quizá esa preeminencia se deba al hecho de que el viejo corpus sigue siendo depositario de principios e institutos definidores de lo que Mortati, y también Bon Valsassina, consideraron la normatividad intrínseca conformadora de una constitución en sentido material o sustancial.

Pero volvamos al reglamento parlamentario, con la convicción de que, como norma, ha de insertarse en un ordenamiento jurídico.

El mérito de Santi Romano, como advierte en la propia doctrina italiana Silvio de Fina, es el de haber vinculado en términos de identidad los conceptos de derecho y de ordenamiento jurídico con el de institución y, al mismo tiempo, el haber subrayado la pluralidad de instituciones dentro del área del Estado. Sin embargo, al reducir a éste al nivel de una mera institución, elevando al rango de ordenamiento jurídico a cualquier ordenamiento social, Romano nos lleva a la imposibilidad de distinguir los ordenamientos estatales de los ordenamientos sociales comunes, y ello por cuanto que, como denunció en la doctrina fascista de los años treinta Sergio Pannunzio, con su posición, Romano aceptaba acríticamente respecto del Estado una condición social común que es desmentida por la realidad.

A nuestro juicio, y siguiendo al maestro italiano a pesar de asumir la crítica apuntada, se impone una redefinición del concepto de ordenamiento jurídico que a su vez consagre en derecho un concepto matizado y, por ende, heterogéneo del Estado.

$\mathrm{La}$ afición de los juristas a «los viejos instrumentos» conceptuales les lleva a veces a confundir rigor científico con comodidad o rutina doctrinal y, en otros casos, a rechazar como espurias aportaciones procedentes de campos ajenos a su disciplina.

A nuestro juicio, sin embargo, nada más contradictorio con el talante humanista que el Derecho imprime que esa actitud.

Así, ante el tema que nos ocupa, entendemos que, entre otras muchas, hay una aportación de la ciencia política que está pendiente de encontrar un eco en el mundo de lo jurídico. Se trata del reconocimiento de los elementos de organización y de soberanía territorial como dualidad definitoria del Estado. Hermann Heller, en formulación ya clásica, lo plantea en estos términos: «El género próximo del Estado es, pues, la organización, la estructura de efectividad organizada en forma planeada para la unidad de la decisión y acción. La diferencia específica con respecto a todas las demás organizaciones es su calidad de dominación territorial soberana.»

Admitida la exactitud de esa formulación, entendemos que debe concluirse 
diferenciando dos manifestaciones del ordenamiento jurídico del Estado: una articulada como ordenamiento jurídico institucional en sentido estricto (en el que se integra el Derecho parlamentario) y otra definida como ordenamiento jurídico territorial concebido como manifestación no tanto de dominio cuanto del imperio y de la competencia que el Estado asume. Y al igual que toda competencia requiere la previa dotación de un órgano para su ejercicio, el ordenamiento institucional constituye un prius respecto del segundo. Al ordenamiento territorial corresponde, como expresión de la acción del Estado, integrar el sistema tradicional de las fuentes del Derecho y los principios jurídicos garantizados por la Constitución (art. 9.3), en cuanto que a él van referidos, y no al nivel institucional prefigurado exclusivamente en la norma fundamental.

Sin embargo, a diferencia de lo que en Derecho medieval supuso la coexistencia de los statuta y del ius commune, lo apuntado hasta ahora presupone y no atenta a la unidad del ordenamiento jurídico; se trata sólo de superar su consideración en términos de univocidad. Su unidad, en realidad, viene garantizada, aunque no definida, por la articulación del principio de soberanía. Cabe destacar, de otra parte, que la unidad del ordenamiento jurídico, en su manifestación como sistema, aflora como exigencia primaria en toda teoría de la interpretación de las leyes. Así, al insertar el reglamento parlamentario en el ordenamiento jurídico por la vía institucional antes expuesta, consideramos que ello conlleva la sujeción de su virtualidad, al igual que ocurre con toda norma, a los principios jurídicos generales sobre aplicación y eficacia de las normas jurídicas (así, los contenidos en el título preliminar del Código Civil), salvo en cuanto quede excluido por su fin o ratio specialis.

Una manifestación nítida de la inserción del reglamento parlamentario en el ordenamiento jurídico del Estado la encontramos en la Ley Orgánica 2/1979, de 3 de octubre, reguladora del régimen del Tribunal Constitucional. En su artículo veintisiete, apartado dos, se consideran susceptibles de declaración de inconstitucionalidad, entre otras disposiciones normativas, a los Reglamentos de las Cámaras y de las Cortes Generales y a los Reglamentos de las Asambleas Legislativas de las comunidades autónomas. Tiene especial interés la inclusión de estos últimos en el objeto de dicha declaración de inconstitucionalidad, por cuanto que con ello se reconoce la inserción del ordenamiento jurídico estatutario dentro del ordenamiento del Estado, de conformidad con lo dispuesto por el artículo 147, apartado 1, de la Constitución.

El artículo veintiocho, apartado uno, de la misma Ley Orgánica integra al reglamento parlamentario en lo que el Consejo Constitucional francés ha denominado «bloque de constitucionalidad», al disponer que «para apreciar la conformidad o disconformidad con la Constitución de una ley, disposición o acto con fuerza de ley del Estado o de las comunidades autónomas, el Tribunal considerará, además de los preceptos constitucionales, las leyes que dentro del marco constitucional se hubieran dictado para delimitar las competencias del Estado y las diferentes comunidades autónomas o para regular o armonizar el ejercicio de las competencias de éstas». A nuestro juicio, entre esas «leyes» (interpretando el término en sentido lato, como disposición con virtualidad jurídica) que tienen por objeto la delimitación de las competencias 
de los entes territoriales aludidos debe considerarse incluido el reglamento parlamentario, en cuanto que éste contiene normas que inciden directamente sobre el ejercicio y, por tanto, el alcance de tales competencias.

En cuanto a la verificación de la constitucionalidad del propio reglamento parlamentario, sólo cabe su parangón directo con la Constitución, como, desde una mayor rigidez y limitación para la autonomía normativa de las Cámaras, reconoce el artículo 61 de la Constitución francesa vigente; conforme a este precepto, «las leyes orgánicas antes de su promulgación y los reglamentos de las asambleas parlamentarias, antes de ser puestos en vigor, deberán ser sometidos al Consejo Constitucional, el cual se pronunciará sobre la conformidad de unas y otros con la Constitución».

Una vez situado el reglamento parlamentario dentro del marco del ordenamiento jurídico, importa subrayar una diferencia, no por evidente de menor interés. La función estatutaria, y por tanto dirigida primariamente a articular el funcionamiento interno de la institución parlamentaria, no excluye, sino que presupone determinados efectos externos de las normas contenidas en dicha disposición.

La distinción, dentro del reglamento parlamentario de interna corporis y externa corporis, tan lúcidamente matizada por Bon Valsassina en la doctrina italiana, responde a un doble orden de consideraciones.

En primer lugar, respecto del fundamento jurídico de las normas con eficacia externa incluidas en el Reglamento de las Cámaras, entendemos con el autor precitado que, si bien la actividad de éstas está subordinada al ordenamiento jurídico que la Constitución garantiza, la institución parlamentaria actúa no ejecutando, sino observando las reglas instrumentales sobre la creación de normas jurídicas, y desde ese contexto instrumental, y en razón, por tanto, de su función, el Parlamento puede incorporar a su reglamento válidamente disposiciones jurídicas de contenido material. Esto es comúnmente admitido por la doctrina, habiéndose superado posiciones contrarias como la de Santi Romano.

En segundo lugar, es evidente que la garantía de la integridad de la función parlamentaria comporta la existencia de normas de los Reglamentos de las Cámaras que afecten directamente a órganos extraños a las mismas. Baste aludir a la remisión que a los Reglamentos de las Cámaras hace el artículo 87.1 de la Constitución para articular la iniciativa legislativa del Gobierno; o referirnos al régimen de inmunidades parlamentarias e incluso a instrumentos parlamentarios típicos como el ruego, la pregunta, la interpelación o la moción. En todos estos casos, en los que el poder judicial y el Gobierno se ven implicados, la función parlamentaria modula los efectos externos e internos de las normas del reglamento.

\section{III}

El Derecho parlamentario puede definirse, siguiendo a Vincenzo Longi, como el conjunto de normas referidas al Parlamento, considerado como la representación natural de la soberanía popular, de conformidad con el régimen 
establecido por la Constitución y con el principio de colaboración entre los poderes del Estado.

En el enunciado de las fuentes del Derecho parlamentario aludiremos en primer lugar a la Constitución y a las leyes orgánicas $\mathrm{u}$ ordinarias que la desarrollan en cuanto afecten al régimen de las Cámaras; de entre las de carácter orgánico destacaremos en nuestro ordenamiento jurídico la Ley Electoral, cuya trascendencia se pone de relieve con la simple lectura del artículo 70 de aquella norma fundamental. Queda excluido el decreto-ley, por prohibición expresa del artículo 86.1 de la misma Constitución.

En segundo lugar, el reglamento parlamentario, fuente directa de primer orden, representa la aplicación del principio jurídico-político conforme al cual los órganos constitucionales, y en especial los parlamentarios, una vez que la norma fundamental ha definido su función, pueden regular autónomamente el ejercicio de su actividad.

La importancia de la costumbre parlamentaria, aun siendo cardinal para la evolución de toda asamblea legislativa, se ve disminuida en las Cámaras, que, a diferencia de las de influencia anglosajona, adoptan para su gobierno y ejercicio de su función un estatuto orgánico o reglamento parlamentario cuya vigencia no se limita necesariamente a cada legislatura.

No son, a nuestro juicio, fuente del Derecho parlamentario, en sentido formal o directo, las instrucciones o acuerdos de los órganos de gobierno de las Cámaras, si bien éstos pueden considerarse fuente en sentido real o primario. Tales disposiciones, así como el reglamento de régimen interior en su caso, son expresión derivada del propio reglamento parlamentario.

En Derecho común se incluyen también entre las fuentes del Derecho a los principios generales, informadores del ordenamiento jurídico, como reconoce el párrafo 4 del artículo $1 .^{\circ}$ del Código Civil. Admitimos, con Longi, la virtualidad de determinados principios generales específicos del Derecho parlamentario o, más exactamente, expresión del espíritu mismo de todo régimen parlamentario. Así, el principio de la voluntad de la mayoría, que debe tener la posibilidad de manifestarse según el modo previsto en la Constitución; o el principio de la minoría, que debe ser tutelada y que tiene derecho a ejercer la crítica y la oposición dentro de las Cámaras.

Podemos ahora preguntarnos si todo enunciado de fuentes del Derecho conduce, como ya antes hemos apuntado, a la necesidad de aplicar el principio de jerarquía normativa. En el supuesto del Derecho parlamentario entendemos que no, en los términos en que comúnmente se consagra; y ello por cuanto que dicho ordenamiento institucional está íntegramente prefigurado en la Constitución. A esta forma fundamental, como marco de la articulación orgánica de la institución parlamentaria, corresponde la incorporación de otras fuentes por la vía de la remisión normativa; incorporación que, una vez admitida la primacía de la propia Constitución, implica antes convergencia que enumeración jerarquizada.

Dicha remisión constitucional configura supuestos de reserva normativa; valga la remisión a los artículos $79.2,80$ y 86.2 in fine.

Problema de no menor interés a la hora de definir el alcance del reglamento parlamentario es el de su interpretación. En las Cámaras con régimen 
de Presidencia fuerte corresponde de ordinario al titular de esta dicha función; es el caso de nuestro Congreso de los Diputados, de conformidad con el artículo 23 de su Reglamento provisional, a cuyo tenor el presidente «cumple y hace cumplir el Reglamento, interpretándolo y supliéndolo en casos de duda u omisión». Distinto es el pronunciamiento del Reglamento de la Cámara Alta, y así, en su artículo 28, se reconocen al presidente del Senado, entre otras, dos facultades distintas: «8. Interpretar el Reglamento», y «9. Suplir, de acuerdo con la Mesa de la Comisión del Reglamento, las lagunas de éste.» Tras esta dualidad subyace la diferencia entre la virtualidad actual del reglamento como norma de gobierno de un órgano en activo y la virtualidad potencial o institucional del mismo. Nada más justificado que esta segunda dimensión normativa conlleve una interpretación del reglamento de mayor rigor institucional. Así se reconoce en otros Parlamentos, como es el caso de la Cámara de los Diputados italiana, en la que cumple esa función la Junta del Reglamento (Giunta del Regolamento).

Un mayor rigor institucional en la interpretación del reglamento parlamentario debiera conducir al reforzamiento de su estabilidad normativa, evitando que los programas o calendarios de gobierno de las Cámaras impliquen derogaciones singulares del mismo, reduciéndolo a mera subsidiariedad residual. En ello están en juego no sólo los principios de legalidad y de interdicción de la arbitrariedad de los poderes públicos, sino incluso la virtualidad de la propia institución parlamentaria, primariamente vinculada al reglamento (véanse artículos $67.3,79.1,87.1,89.1$ y 111.1, entre otros).

No puede desconocerse que el mayor enemigo del Parlamento es el arbitrismo en cuanto generador de un clima de provisionalidad institucional. Sólo el respeto, que no la rígida veneración del reglamento, puede dar sentido y coherencia institucional a las Cámaras.

La edad de la descodificación, en expresión feliz de Irti, pudiera ser, a nuestro juicio, no sólo un tiempo de crisis de los viejos códigos liberales, gestados al calor del optimismo racionalista burgués, sino también un tiempo de quiebra de los procesos institucionales de creación de normas jurídicas. Y, podríamos añadir, ¿no supone ello una quiebra del concepto mismo de Derecho?... La Constitución, como código político, heredera de la vocación de coherencia de los viejos códigos civiles, se nos presenta como la garantía normativa de la unidad del ordenamiento jurídico.

Como articulación directa de esa norma constitucional, el reglamento parlamentario debiera garantizar, de otra parte, la autonomía institucional de las Cámaras respecto de los partidos políticos. Estos se integran en aquéllas a través de los grupos parlamentarios no como partidos, sino como articulación ideológica del cuerpo electoral representado. La coherencia con este principio hace aconsejable, a nuestro juicio, la necesidad de homologar institucionalmente el estatuto de todos los grupos parlamentarios para evitar que se convierta en el mero estatuto de los partidos presentes en el Parlamento. Los partidos pueden llegar a controlar el proceso electoral y, como reconoce el artículo 6 de la Constitución, «son instrumento fundamental para la participación política», pero en las Cámaras no tienen la condición de sujeto representado que asume el pueblo en exclusiva (véase artículo 66.1). 
Sólo esa hipóstasis subsuntiva de los partidos políticos en la institución parlamentaria (gracias a la cual, por otra parte, vieron históricamente reconocida su dimensión constitucional; es el caso de los parliamentary parties en el Derecho inglés o incluso, mucho más tarde, entre nosotros las «fracciones políticas», en el Reglamento de 29 de noviembre de 1934), sólo gracias a su reconocimiento de la función política de las Cámaras podrá superarse la vexata questio de la crisis del Parlamento e incluso de la crisis del Estado, comúnmente definida como fruto de la sustitución del poder del Estado por el de los grupos.

Sin embargo, finalmente, debemos reconocer que la vigencia real del reglamento parlamentario, como la de la propia Constitución, es el efecto y no la causa de una conciencia institucional definida. Porque entendemos, siguiendo a Longi, que el régimen parlamentario, más que la suma de una serie de garantías constitucionales escritas y de normas sobre el funcionamiento sobre órganos y poderes que califican a un Estado como parlamentario, consiste más bien en un ambiente, en una atmósfera en la cual se desarrolla, de acuerdo con los principios constitucionales, un Estado libre a través de sus instituciones fundamentales, y sobre todo a través del Parlamento. Un régimen político de libertades es indispensable para conferir valor sustancial al propio Derecho parlamentario, ya que éste no puede considerarse - añade Longi- como un mero complejo de normas rígidas que regulan la actividad de un Parlamento cualquiera.

Y terminamos. El Parlamento, ayer como hoy, sólo puede caminar en dos direcciones: o en la de recoger el pálpito real del ser y del sentir del pueblo representado o en la de convertirse en un estéril y prosaico «salón de los pasos perdidos».

\section{BIBLIOGRAFIA}

1. Alzaga Villanmil, O.: Contribución al estudio del Derecho parlamentario, en «Revista de Derecho Público», 2.a época, núm. 6, Madrid, 1976.

2. Biscaretti di Ruffia, P.: Derecho constitucional, Edit. Tecnos, Madrid, 1973.

3. Bon V.alsassina, M.: Sui Regolamenti parlamentari, Padua, 1955.

4. De Castro y Bravo, F.: Derecho civil de España, t. I, Instituto de Estudios Políticos, Madrid, 1955.

5. DE FINA, S.: Stato e Istituzione. Saggio per una revisione critica della teoria istituzionale del Diritto, Milán, Dott. A. Giuffrè, ed. 1974. Diritto e Società, idéntica editorial y año de la obra.

6. GarCía DE EnTERría, E., y Fernández, T. R.: Curso de Derecho administrativo, I, Ed. Civitas, Madrid, 1977.

7. Heller, H.: Teoría del Estado, Fondo de Cultura Económica, México, 1974.

8. LongI, V.: Elementi di Diritto e Procedura Parlamentare, Milán, Dott. A. Giuffrè, edición 1978.

9. Pérez Serrano, N.: Naturaleza juridica del reglamento parlamentario, en «Revista de Estudios Políticos», núm. 105, pág. 103.

10. Romano, S.: L'Ordre juridique, Dalloz, París, 1975.

Fragmentos de un diccionario jurídico, Ediciones Jurídicas Europa-América, Buenos Aires, 1964. 